\title{
ANÁLISE DA QUALIDADE DA SIMULAÇÃO DO FLUXO DE AR DE CHAMINÉS SOLARES COM O ENERGYPLUS
}

\section{ANALYSIS OF THE QUALITY SOLAR CHIMNEY SIMULATIONS USING ENERGYPLUS}

\author{
Matheus Menezes Oliveira ${ }^{1}$ \\ Universidade Federal de Viçosa, Viçosa, MG, Brasil, matheus.meneses@gmail.com \\ Joyce Correna Carlo ${ }^{2}$ \\ Universidade Federal de Viçosa, Viçosa, MG, Brasil, joycecarlo@ufv.br
}

\section{Resumo}

A ventilação é uma das principais estratégias para alcançar conforto e eficiência energética em climas quentes e úmidos. Nesse contexto, as chaminés solares são dispositivos que promovem o aumento das trocas de ar no ambiente e redução dos gastos energéticos de um edifício. Vários autores utilizaram simulações termoenergéticas em seus estudos sobre o tema, entretanto, não foram encontrados na literatura parâmetros referenciais para uma avaliação numérica anual dos fluxos de ar de uma chaminé solar. Esse artigo apresenta uma investigação sobre três métodos de simulação de chaminés solares no EnergyPlus: modelo ThermalChimney, Airflow Network e HorizontalOpenings. Os métodos foram discutidos em relação à modelagem e suas restrições, com identificação das vantagens e desvantagens de cada. Os fluxos de ar foram comparados àqueles de um modelo numérico desenvolvido por $C F D$ em estudo anterior que, por sua vez, foi validado com medições em um protótipo construído. Para o tratamento dos fluxos de ar, foram utilizados três indicadores estatísticos, Erro Absoluto Médio, Erro Quadrático Médio e Diferenças Médias, em que o melhor resultado foi encontrado para o método ThermalChimney, cujo Erro Absoluto Médio foi entre 37\% e 52\% em comparação aos demais, que variaram de $65 \%$ a $229 \%$. Estes resultados preenchem uma lacuna na literatura ao indicar algumas diretrizes para simulação e, em especial, por analisar indicadores para a comparação entre fluxos de ar de simulações termoenergéticas de chaminés solares com o EnergyPlus.

Palavras-chave: Chaminé Solar. Simulação Termoenergética. EnergyPlus.

\begin{abstract}
Natural ventilation is one of the most important strategies for achieving comfort and energy efficiency in hot and humid climates. In this framework, the solar chimneys are devices that promote the increase of air changes in the room and reduction of the energy consumption of buildings. Several authors have used energy performance simulations in their studies regarding this issue. However, reference parameters for an annual numerical assessment of the air flow promoted by a solar chimney were not found in the literature. This article presents an investigation of three methods of simulation of solar chimneys in EnergyPlus: model ThermalChimney, Airflow Network, and HorizontalOpenings. The methods were discussed regarding modeling and its restrictions, with the statement of the advantages and disadvantages of each approach. The airflow simulation results were compared to a numerical model developed through CFD in a previous study, validated with measurements in a constructed prototype. Three statistical indicators, to treat the airflow, were used: Mean Absolute Error, Mean Square Error and Mean Differences. The best result was found for the thermal chimney model, with Average Absolute Error between $37 \%$ and $52 \%$ in comparison to the others, which floated from $65 \%$ to $229 \%$. These results fill a gap in the literature by indicating some guidelines for simulation, and in particular, by analyzing indicators for the comparison between air flows resulting from energy performance simulations of solar chimneys with EnergyPlus.
\end{abstract}

Keywords: Solar chimney. Thermal and Energy Simulation. EnergyPlus.

\section{How to cite this article:}

OLIVEIRA, Matheus Menezes; CARLO, Joyce Correna. Análise da qualidade da simulação do fluxo de ar de chaminés solares com o EnergyPlus. PARC Pesquisa em Arquitetura e Construção, Campinas, SP, v. 9, n. 2, p. 86-96, jun. 2018. ISSN 1980-6809. Disponível em: <https://periodicos.sbu.unicamp.br/ojs/index.php/parc/article/view/8650284>. Acesso em: 20 maio 2018. doi:https://doi.org/10.20396/parc.v9i2.8650284. 


\section{Introdução}

Em climas quentes e úmidos, a ventilação é uma das principais estratégias para alcançar conforto e eficiência energética. O resfriamento passivo, segundo Imran, Jalil e Ahmed (2015), desempenha um papel importante para proporcionar um ambiente termicamente adequado para o conforto humano. A ventilação natural pode ocorrer através da ação direta dos ventos, do efeito chaminé ou da combinação de ambos. A mesma, segundo Schulze e Eicker (2013), deve atender a duas funções principais: proporcionar qualidade e trocas do ar interno, sem a necessidade do uso de eletricidade e aumentar o nível de conforto em dias quentes. Entretanto, de acordo com os autores, a falta de estudos e planejamento de aberturas para a ventilação natural, assim como a pouca disponibilidade de resultados de avaliações de edifícios ventilados naturalmente, prejudica a aplicação desse tipo de estratégia.

Uma das formas de aumentar a movimentação de ar no interior de um edifício em área urbana, em que existem grandes perdas da velocidade do vento devido aos obstáculos, é através da impulsão térmica. Essa estratégia é incrementada pela radiação solar, em que o ar aquecido ascende devido à diferença de temperatura e pressão entre o meio interno e externo (NEVES; RORIZ, 2012). Uma das formas de promover a impulsão térmica é através de chaminés solares, dispositivos que, embora não dependam da ação direta dos ventos, sofrem efeitos de sua influência.

As chaminés solares possuem grande potencial para o aumento das renovações de ar em edificações, não geram ruídos, possuem um baixo custo operacional e não necessitam de energia elétrica (ASADI et al., 2016). Por causa dessas características, esses dispositivos têm despertado interesse de projetistas devido a sua longa vida útil e economia para edificações (SHI et al., 2016). Zhai, Song e Wang (2011), Asadi et al., (2016) e Hosien e Selim (2017), as definem como um sistema de termossifão solar que melhora a qualidade interna e aumenta as taxas de renovação do ar através do efeito chaminé.

As chaminés solares são semelhantes às chaminés convencionais, exceto pelo princípio de captar a radiação solar para aumentar o efeito chaminé a partir de uma superfície externa envidraçada, voltada para a orientação que permita maior incidência de radiação. Essa radiação é transmitida pelo vidro, é absorvida pela superfície oposta e aquece o ar no interior da cavidade por radiação e convecção. As parcelas de absorção e reflexão variam de acordo com as propriedades do vidro utilizado.

Uma parcela da radiação solar transferida para a cavidade é absorvida pela placa absorvedora e o calor é transferido por convecção ao ar no interior da cavidade, contribuindo para o aumento de sua temperatura. $\mathrm{O}$ ar aquecido na cavidade se expande, se torna menos denso, e tende a ascender ao topo da chaminé, através do efeito chaminé (ASADI et al., 2016).

Maciel (2016), Neves e Silva (2017) e Shi e Zhang (2016), apontam que a relação entre a quantidade de radiação solar direta incidente no dispositivo e o consequente aumento da temperatura superficial da placa absorvedora são fatores essenciais para aumentar as renovações de ar no interior do ambiente e a eficiência do dispositivo.

Nesse contexto, este trabalho tem por objetivo avaliar diferentes métodos de simulação de uma chaminé solar no EnergyPlus por meio de indicadores estatísticos para comparação dos fluxos de ar para todas as horas do ano.

\section{Simulação termoenergética de chaminés solares}

Métodos de análise foram desenvolvidos e testados para prever o comportamento e efeitos do vento em edificações devido a sua importância e complexidade. Esses modelos têm como objetivo, além de prever o desempenho da ventilação, fornecer informações relativas a parâmetros do ar no interior de um ambiente ou em um edifício, antes mesmo da sua construção ou reforma (CHEN, 2009). Entre os métodos de análise de dispositivos proporcionadores de ventilação natural, destacam-se na literatura os modelos analíticos, modelos experimentais em escala, modelos em escala 1:1, modelos multizonas (EnergyPlus) e modelos CFD (Computational Fluid Dynamics).

Nesse contexto, a pesquisa de Maciel (2016), utilizou três métodos de análise simultaneamente para avaliar um modelo de chaminé solar. A autora propôs um dispositivo que mescla as características de uma proteção solar convencional (brise) e uma chaminé solar (Figuras 1, 2 e 3 ), que resultou no depósito de patente do produto denominado Brise-Chaminé-Solar. O desempenho do Brise-Chaminé-Solar foi analisado através de simulação termoenergética nos programas ANSYS CFX e EnergyPlus e de medições em modelo construído em escala 1:1 Por este método, Maciel (2016) comprovou que o Brise-Chaminé-Solar apresenta potencial para a renovação de ar e redução do ofuscamento em ambientes internos. A autora destaca que, para maximizar a eficiência do dispositivo quanto à ventilação natural, o mesmo deve ser conectado a apenas um pavimento, sempre que possível.

Outros pesquisadores, como Lee e Strand (2009), Neves e Roriz (2012), Koronaki (2013), Asadi et al. (2016) e Neves e Silva (2017) realizaram seus estudos a partir de simulações no EnergyPlus e alcançaram resultados satisfatórios na simulação de diferentes chaminés solares. 
Figura 1 - Seção horizontal do Brise-Chaminé-Solar. (a) Dimensões e inclinação com a parede (b) Conexão entre o Brise-Chaminé-Solar e o ambiente interno
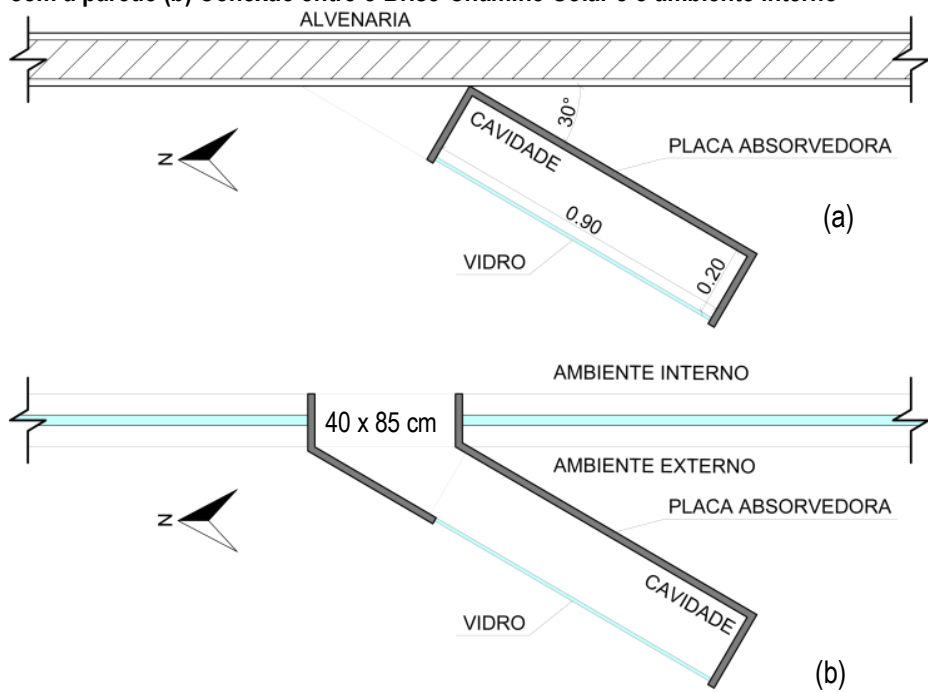

Fonte: Adaptado de Maciel (2016).

Figura 2 - Perspectiva isométrica do Brise-Chaminé-Solar. (a esquerda) Frente (a direita) Fundos

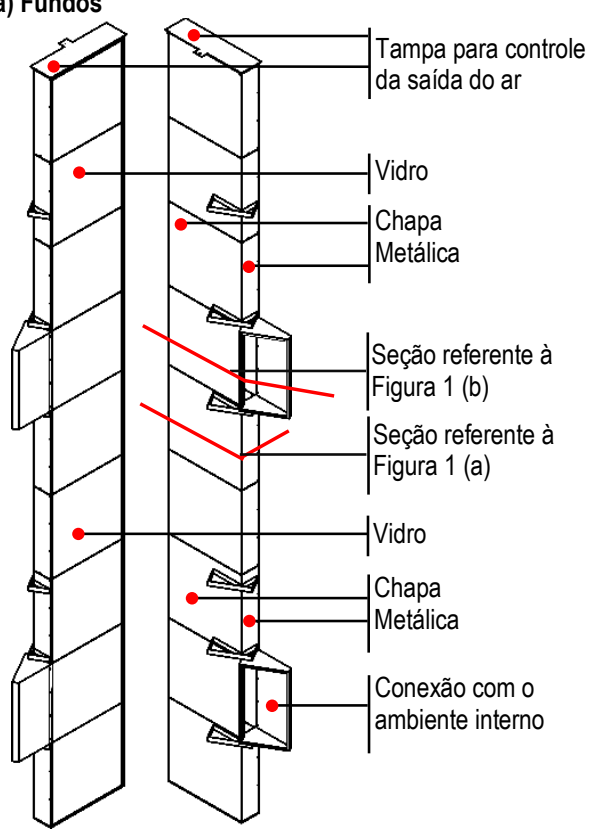

Fonte: Adaptado de Maciel (2016).

O EnergyPlus é um programa computacional criado a partir dos programas $B L A S T$ e $D O E-2$ disponibilizado pelo Departamento de Energia dos Estados Unidos e desenvolvido para análise energética de edificações, para simulação de cargas térmicas, temperatura, ventilação, radiação entre outros (MELO; WESTPHAL; MATOS, 2009). O programa utiliza o método de cálculo do balanço térmico, validado pelo método bestest da ASHRAE 140 (ASHRAE, 2011) e suas simulações termoenergéticas utilizam o modelo de rede para o cálculo do fluxo de ar, denominado AirflowNetwork. De acordo com Zhang, Lam e Yao (2013), no modelo de rede adotado pelo programa, cada zona é um nó do sistema, em que as aberturas são ligações entre esses nós. Neves e Roriz (2012) apontam que este modelo se baseia na hipótese de que as temperaturas superficiais e do ar são uniformemente distribuídas dentro de uma mesma zona térmica.

Figura 3 - Corte Esquemático Ambiente/Brise Chaminé Solar

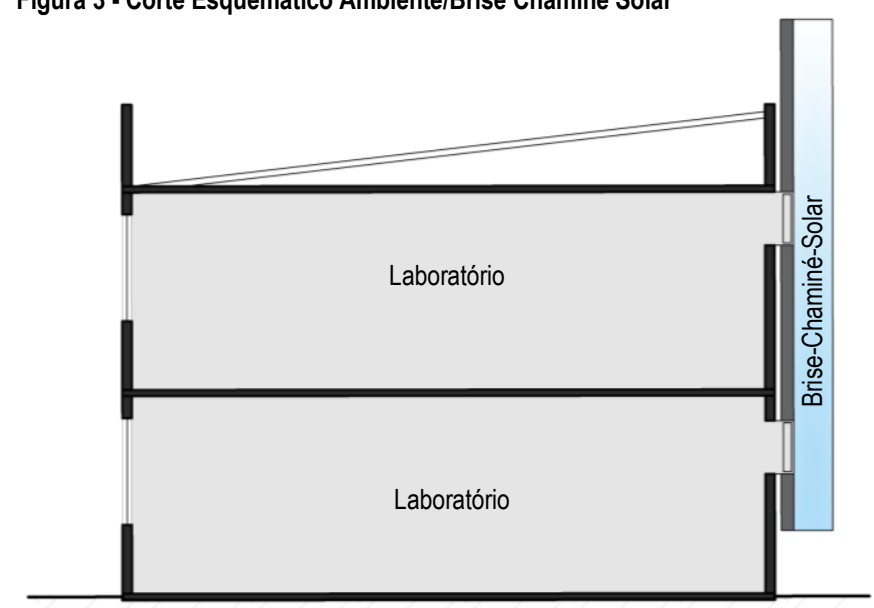

Fonte: Os autores.

Para simulações de chaminés solares, o programa recomenda o método de modelagem denominado ThermalChimney. Nesse método, o algoritmo utilizado para o balanço energético é semelhante ao utilizado para o cálculo da parede Trombe, em que é levado em consideração a temperatura da placa absorvedora e a cobertura de vidro. Entretanto, a distribuição vertical da temperatura do ar e a temperatura de descarga resultantes da chaminé térmica são calculadas a partir de algoritmo próprio para chaminé térmica (ENERGYPLUS, 2015).

Os modelos numéricos de transferência de massa do EnergyPlus, especialmente no sentido ascendente, podem ser mais investigados com o uso de resultados experimentais e teste de diferentes opções de modelagem e simulação no próprio programa. Essas diferentes opções necessitam de calibração com outro tipo de análise complementar, e suas diferenças estabelecidas por indicadores estatísticos.

Em seus estudos, Lee e Strand (2009) e Asadi et al., (2016) utilizaram o EnergyPlus para simular uma chaminé solar. Os autores adotaram testes encontrados em outros estudos sobre chaminés solares para validação dos fluxos de ar. Porém, não foi estabelecida uma relação entre os fluxos obtidos por simulação com as referências para a validação.

Neves e Roriz (2012), Neves e Silva (2017) e Asadi et al., (2016) avaliaram os fluxos de ar de uma chaminé solar por apenas um dia. Neves e Roriz (2012) e Neves e Silva (2017) apresentaram uma comparação entre a simulação no EnergyPlus com modelos matemáticos e testes em um protótipo construído, estabelecendo uma diferença entre as médias diárias de fluxo de ar de 7\%. Koronaki (2013), também avaliou os fluxos de ar de uma chaminé solar por 
um dia. $\mathrm{O}$ autor apresentou uma comparação entre a simulação no EnergyPlus com medições realizadas em um protótipo construído. As diferenças entre os fluxos simulados e medidos foram de $10 \%$.

Embora citados diversos autores que utilizaram o EnergyPlus para a simulação do efeito chaminé, não foram encontrados na literatura parâmetros referenciais para uma avaliação numérica anual dos fluxos de ar de uma chaminé solar, visto que somente com a simulação anual é possível produzir uma avaliação completa da aplicabilidade desses dispositivos (LEE; STRAND, 2009). Nos estudos supracitados, o método de modelagem adotado no EnergyPlus para a simulação de chaminés solares é descrito de maneira simplificada, e não foram apresentados os métodos comparativos utilizados para validar os modelos simulados.

\section{Método}

O método utilizado nesse trabalho é baseado na comparação de diferentes simulações termoenergéticas com o software EnergyPlus, tendo como referência o dispositivo Brise-Chaminé-Solar desenvolvido por Maciel (2016). Os fluxos de ar obtidos por simulações por três métodos são comparados aos fluxos referenciais do BriseChaminé-Solar através de indicadores estatísticos.

\section{Caracterização do Brise-Chaminé-Solar}

O Brise-Chaminé-Solar possui uma geometria retangular com dimensões internas de $0,90 \mathrm{~m}$ por $0,20 \mathrm{~m}, 8,00 \mathrm{~m}$ de altura total e a conexão entre o dispositivo e o ambiente possui $0,40 \mathrm{~m}$ por $0,85 \mathrm{~m}$, de acordo com as Figuras 1, $2 \mathrm{e}$ 3.

O protótipo foi instalado no edifício anexo ao Departamento de Arquitetura e Urbanismo (DAU), localizado na Universidade Federal de Viçosa, em Viçosa, Minas Gerais (Figura 4). A cidade está localizada na latitude $20,77^{\circ} \mathrm{Sul}$ e longitude $42,87^{\circ}$ Oeste e a $712,2 \mathrm{~m}$ acima do nível do mar.

Segundo Maciel (2016), o dispositivo tem como características construtivas:

(...) uma placa vertical composta de múltiplas camadas: uma superfície envidraçada, uma cavidade por onde o ar flui e uma placa absorvedora. A placa absorvedora é composta por uma chapa de aço na cor preta (alta absortância térmica), uma camada de ar para isolamento térmico e outra chapa de aço para fechamento. A face externa da chapa de fechamento, voltada para a abertura do ambiente interno, tem cor de baixa absortância solar e da luz visível e tem a função de refletir a radiação externa, bem como bloquear a radiação térmica emitida pela placa absorvedora. (p.28).
Figura 4 - Brise-Chaminé-Solar. (a direita) Vista Geral (a esquera) Superfície Refletora

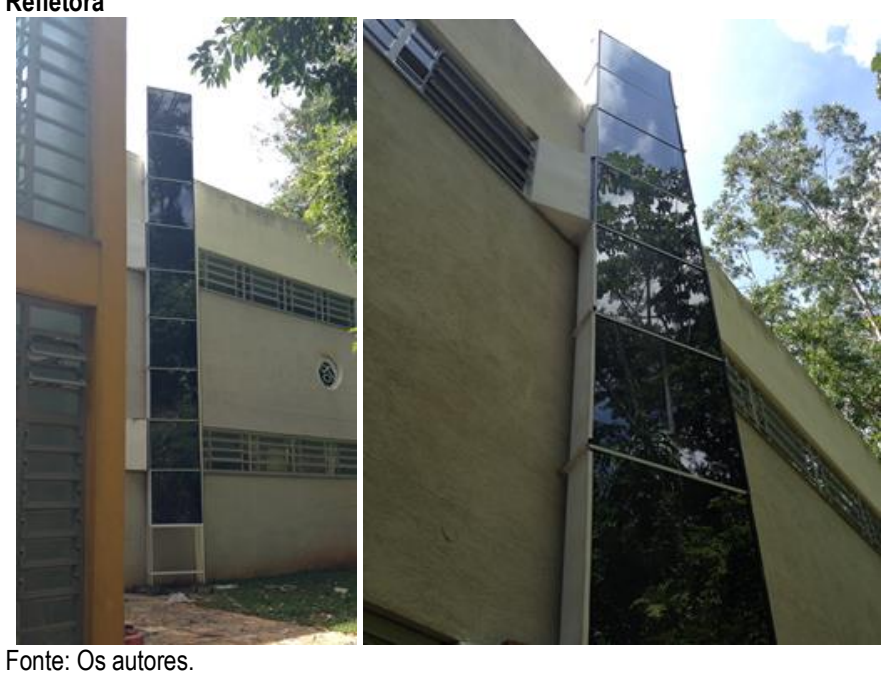

\section{Fluxos de ar referenciais}

As equações de Maciel (2016) para estimativa do fluxo de ar foram obtidas por simulação em $C F D$ e validadas pela autora por meio de medições no protótipo mostrado na Figura 4. O dispositivo foi acoplado em dois laboratórios: o primeiro, um ambiente térreo, com área de $107,8 \mathrm{~m}^{2} \mathrm{e}$ volume de $339,6 \mathrm{~m}^{3}$; o segundo, localizado no pavimento superior, com área de $91,3 \mathrm{~m}^{2}$ e volume de $287,6 \mathrm{~m}^{3}$ (Figura 3). O Brise-Chaminé-Solar foi instalado na fachada oeste e encontra-se conectado aos dois ambientes simultaneamente.

Em seguida, Maciel (2016) desenvolveu duas equações por regressão, com base na simulação $C F D$ para duas alturas: 2,85 m (representando um pavimento) e $5,85 \mathrm{~m}$ (dois pavimentos), apresentadas na Figura 5. Para cada altura, cinco condições de temperatura superficial da placa absorvedora foram consideradas $\left(25^{\circ} \mathrm{C}, 45^{\circ} \mathrm{C}, 65^{\circ} \mathrm{C}, 85^{\circ} \mathrm{C}\right.$ e $105^{\circ} \mathrm{C}$ ) para obtenção de fluxos de ar como dados de saída. Os fluxos obtidos por estas equações apresentaram elevada correlação com a simulação CFD, com coeficientes de determinação 0,9956 e 0,9929, e foram considerados válidos para o Brise-Chaminé-Solar e, consequentemente, referências para o presente estudo.

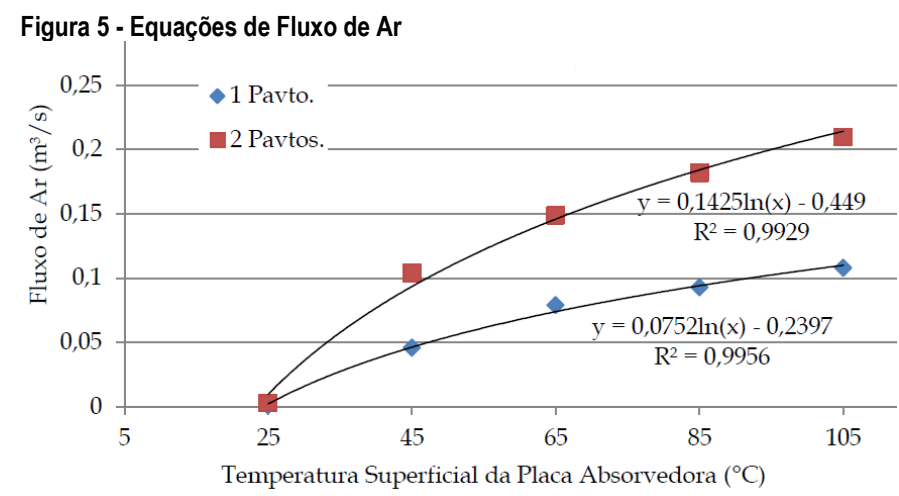

Fonte: Adaptado de Maciel (2016) 


\section{Simulação EnergyPlus}

Para analisar os métodos de simulação, o Brise-ChaminéSolar foi simulado no EnergyPlus, versão 8.4. A simulação foi feita para a cidade de Viçosa-MG, Zona Bioclimática 3, ZB3 (ABNT, 2005) com o arquivo climático TMY desenvolvido por Guimarães e Carlo (2011). A simulação levou em consideração todas as horas do ano e desconsiderou cargas internas de pessoas, equipamentos e condicionamento artificial, conforme condições de contorno estabelecidas por Maciel (2016) para simulações em $C F D$.

O dispositivo foi simulado de três formas distintas: (1) utilizando o modelo de cálculo de chaminé térmica do EnergyPlus, ThermalChimney; (2) utilizando zonas térmicas independentes por pavimento para compor o Brise-Chaminé-Solar, conectadas entre si,
AirflowNetwork; e (3) utilizando o método de zonas térmicas combinadas, HorizontalOpening. Nos dois primeiros métodos de simulação, o Brise-Chaminé-Solar foi modelado para duas alturas, denominadas ALT1 (altura da chaminé equivalente a um pavimento) e ALT2 (altura da chaminé equivalente a dois pavimentos). O método zonas térmicas combinadas não permite a modelagem do dispositivo para ALT1, logo, para esse método de simulação, apenas ALT2 foi avaliada. As propriedades dos materiais dos ambientes em que o dispositivo está acoplado, assim como os materiais do Brise-ChaminéSolar, obstruções do entorno e orientação solar são idênticos aos especificados por Maciel (2016) (Tabela $1 \mathrm{e}$ Figura 6). Foram utilizados como coeficiente de descarga das esquadrias o valor de 0,6 e do Brise-Chaminé-Solar 0,57, conforme recomendado por Andersen (1995) para chaminés solares.

\begin{tabular}{|c|c|c|c|c|c|c|c|c|}
\hline Zona & $\begin{array}{l}\text { Componente } \\
\text { Construtivo }\end{array}$ & Materiais & $\begin{array}{c}\text { Espessura } \\
\text { Equivalente } \\
(\mathrm{m})\end{array}$ & $\begin{array}{l}\text { Condutividade } \\
\text { Térmica } \\
\text { (W/mK) }\end{array}$ & $\begin{array}{c}\text { Densidade } \\
\left(\mathrm{kg} / \mathrm{m}^{3}\right)\end{array}$ & $\begin{array}{c}\text { Calor } \\
\text { Específico } \\
(\mathrm{J} / \mathrm{kgK})\end{array}$ & $\begin{array}{l}\text { Resistencia } \\
\text { Térmica } \\
\left(\mathrm{m}^{2} \mathrm{~K} / \mathrm{W}\right)\end{array}$ & $\begin{array}{c}\text { Fator Solar do } \\
\text { Vidro }\end{array}$ \\
\hline \multirow{8}{*}{ Laboratórios } & \multirow{3}{*}{ Paredes } & Tijolo & 0,140 & 0,90 & 2290 & 920 & 0,016 & \multirow{3}{*}{ - } \\
\hline & & Reboco & 0,030 & 1,15 & 2000 & 1000 & 0,022 & \\
\hline & & Câmara de $\mathrm{Ar}$ & & & & & 0,160 & \\
\hline & Piso & Concreto & 0,100 & 1,75 & 2400 & 1000 & 0,057 & - \\
\hline & \multirow{3}{*}{ Cobertura } & Forro & 0,030 & 0,05 & 50 & 700 & 0,556 & \multirow{3}{*}{-} \\
\hline & & Telha Fibrocimento & 0,070 & 0,95 & 1900 & 840 & 0,007 & \\
\hline & & Câmara de $\mathrm{Ar}$ & & & & & 0,400 & \\
\hline & Janelas & Vidro & 0,006 & - & - & - & - & 0,8 \\
\hline \multirow{4}{*}{$\begin{array}{l}\text { Brise Chaminé } \\
\text { Solar }\end{array}$} & \multirow{3}{*}{ Placa Absorvedora } & Alumínio & 0,003 & 230 & 2700 & 880 & 0,000 & \multirow{3}{*}{-} \\
\hline & & Isopor & 0,020 & 0,04 & 25 & 1420 & 0,830 & \\
\hline & & Chapa de Aço & 0,003 & 55 & 7800 & 460 & 0,000 & \\
\hline & Superfície transparente & Vidro & 0,006 & - & - & - & - & 0,8 \\
\hline
\end{tabular}

Fonte: Os autores.

Figura 6 - Modelo com entorno e orientação

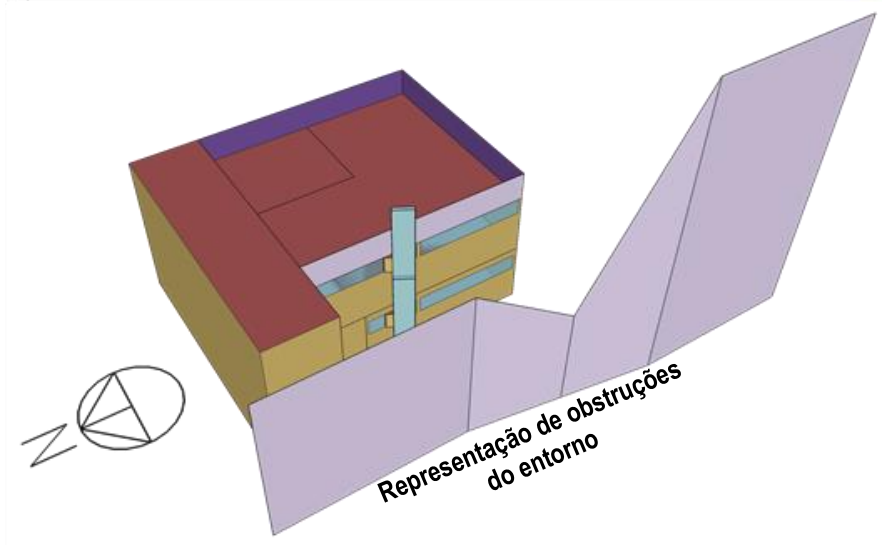

\section{Caso Base}

Foi denominado Caso Base (CB) o fluxo de ar resultante da aplicação das duas equações de Maciel (2016) para ALT1 e ALT2, de acordo com a temperatura superficial horária da placa absorvedora que foi obtida por simulação no EnergyPlus para o ano completo.

\section{Brise-Chaminé-Solar modelado como chaminé térmica (ThermalChimney)}

O Brise-Chaminé-Solar foi modelado como uma chaminé térmica utilizando o modelo de cálculo ThermalChimney (Figura 7), que é recomendado pelo EnergyPlus para esse tipo de simulação. O parâmetro de saída desse modelo é baseado no aumento da taxa de ventilação natural causada pela presença de uma chaminé térmica convencional (Equação 1). Para determinar o aumento da ventilação, a temperatura de descarga do ar no interior da chaminé é calculada com base nas informações sobre a temperatura da placa absorvedora, temperatura da cobertura de vidro e distribuição vertical da temperatura do ar no interior da chaminé (ENERGYPLUS, 2015). 
Figura 7 - Ambiente simulado e Brise-Chaminé-Solar modelado como ThermalChimney no EnergyPlus. (a) Edificação (b) Detalhe da modelagem do Brise-Chaminé-Solar

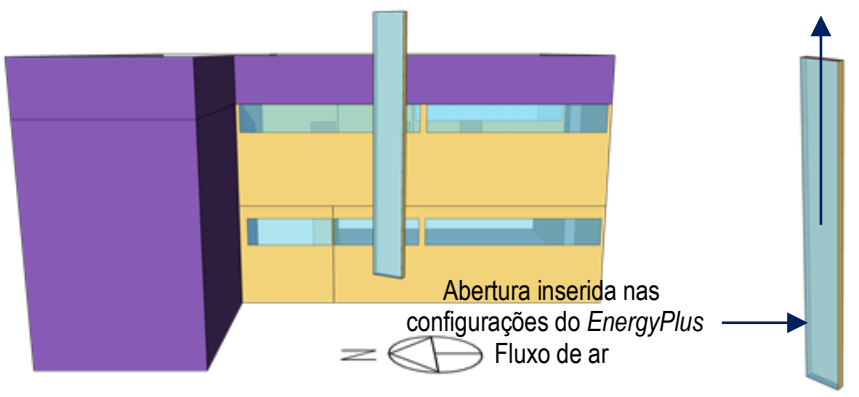

Fonte: Os autores.

$$
\begin{aligned}
& Q=C_{d} A_{o} \sqrt{\frac{2\left(\frac{T_{f o}-T_{r}}{T_{r}}\right) g L}{\left(1+A_{r}\right)^{2}}} \\
& A_{r}=A_{o} / A_{i}
\end{aligned}
$$

Equação 1

Onde: Q - Taxa de fluxo de ar causado pelo efeito chaminé $\left[\mathrm{m}^{3} / \mathrm{s}\right] ; \mathrm{Ai}$ - Área da seção transversal da entrada do canal de ar [m²]; Ao - Área da seção transversal da saída do canal de ar [ $\left.\mathrm{m}^{2}\right] ; \mathrm{Cd}$ - Coeficiente de descarga [adimensional]; $\mathrm{g}$ - Aceleração da gravidade $\left[9,8 \mathrm{~m} / \mathrm{s}^{2}\right] ; \mathrm{L}$ - Altura total da chaminé Solar [m]; Tfo - Temperatura do ar na saída da chaminé solar $[\mathrm{K}] ; \mathrm{Tr}$ - Temperatura do ambiente $[\mathrm{K}]$.

Devido ao fato do modelo de chaminé térmica não se integrar com o modelo de ventilação de rede, foi realizada uma simulação prévia com as aberturas fechadas, com o modelo de rede ativado e sem a chaminé térmica. A partir dessa simulação, obteve-se a infiltração horária, que foi inserida em uma nova simulação com modelo de rede desativado e a chaminé térmica ativada.

\section{Brise-Chaminé-Solar modelado como zonas térmicas conectadas}

O Brise-Chaminé-Solar foi modelado como duas zonas térmicas independentes conforme altura correspondente a cada pavimento, e conectadas entre si através de janelas $100 \%$ do tempo abertas pelo AirflowNetwork (Figura 8). Esse método de simulação não considera o cálculo do efeito chaminé, mas sim a diferença de pressão, logo, é esperado que seus resultados se distanciem do caso base. No entanto, a simulação como zonas térmicas conectadas foi executada como um caso de controle para verificar a escala dos indicadores estatísticos a partir de dois métodos, um recomendado pelo EnergyPlus para o cálculo do efeito chaminé (ThermalChimney) e outro que sabe-se que é incorreto.

Para esse método foram testadas duas alternativas: (1) com as janelas dos ambientes em que o dispositivo está instalado $100 \%$ do tempo abertas; e (2) com as janelas $100 \%$ do tempo fechadas. Nas duas opções, a infiltração foi definida pelo modelo de ventilação de rede, que foi testado anteriormente.

Figura 8 - Ambiente simulado e Brise-Chaminé-Solar modelado como zonas térmicas conectadas no EnergyPlus. (a) Edificação (b) Detalhe da modelagem do Brise-Chaminé-Solar

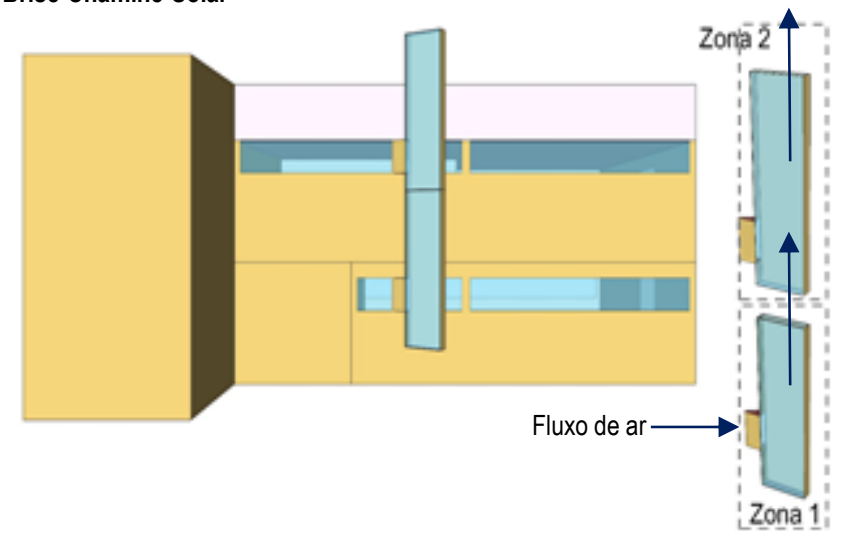

Fonte: Os autores.

\section{Brise-Chaminé-Solar modelado como zonas combinadas (HorizontalOpening)}

O método de zonas térmicas combinadas é recomendado pelo EnergyPlus para simular fluxos de ar em grandes aberturas verticais. Esse método combina fluxos de ar forçado e natural em conjunto e considera que a diferença de pressão a partir da abertura é função da altura, e esta, é resultado da diferença entre a abertura mais baixa e a mais alta. Neste método, a diferença de pressão em toda a zona, assim como a densidade do ar, permanece constante, de modo que é possível apenas calcular o fluxo unidirecional (ENERGYPLUS, 2015).

Embora este método de simulação não seja diretamente recomendado pelo EnergyPlus para a simulação de chaminés solares, sua utilização se justifica por avaliar parâmetros inerentes a chaminés solares, como a diferença de pressão em função da distância e do tamanho das aberturas. Além disso, este método integra-se ao modelo de ventilação de rede, o que pode ser uma simplificação viável em simulações complexas onde o efeito chaminé é apenas mais uma opção de ventilação, dentre a natural e a mecânica. Assim, optou-se por avaliar este método para quantificar as diferenças de fluxo de ar em relação ao modelo de chaminé térmica.

A modelagem consiste basicamente em duas zonas em que o teto da Zona 1 está acima da Zona 2, o ar flui do interior do ambiente para a Zona 1, da Zona 1 para a Zona 2, através de uma abertura que as conecta pelo HorizontalOpening, e da Zona 2 para o exterior (Figura 9). Nesse método, de forma semelhante à simulação de zonas térmicas conectadas, a infiltração foi definida pelo modelo de ventilação de rede. 
Este modelo é válido somente com duas zonas sobrepostas verticalmente, logo não foi possível simular o fluxo de ar do Brise-Chaminé-Solar para a ALT1.

Figura 9 - Ambiente simulado e Brise-Chaminé-Solar modelado como HorizontalOpening no EnergyPlus. (A) Edificação (B) Detalhe da modelagem do Brise-Chaminé-Solar

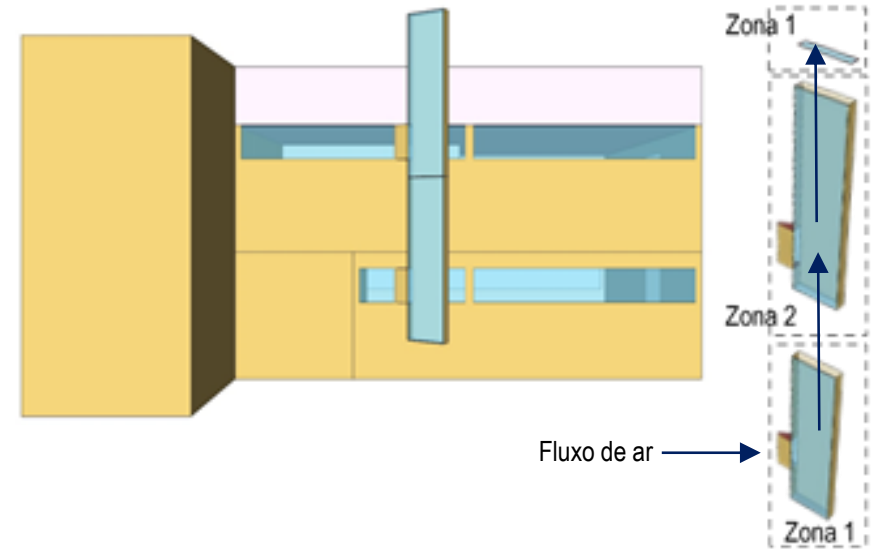

Fonte: Os autores.

\section{Análise dos resultados}

O fluxo de ar simulado com o EnergyPlus para o BriseChaminé-Solar nos três métodos apresentados foi comparado com o resultado do fluxo de ar obtido no caso base.

Para determinar a relação entre fluxos de ar obtidos nas simulações e no caso base, foi utilizado o Erro Absoluto Médio (Equação 2) e Erro Quadrático Médio (Equação 3) e a Diferença Horária Média (Equação 4).

$$
E A M=\sqrt{\frac{\sum_{I}^{N}\left(\frac{y_{i}-x_{i}}{x_{i}}\right)^{2}}{N}} 100
$$

Equação 2

Onde: EAM: Erro Absoluto Médio; $y_{i}=$ Dados obtidos por simulação; $x_{i}=$ Dados obtidos a partir do caso base; $\mathrm{N}=$ número de valores analisados, horas do ano.

$\operatorname{EQM}\left(\theta^{\prime}\right)=\left(\theta^{\prime}-\theta\right)^{2} / \mathrm{N}$

Equação 3

Onde: EQM: Erro Quadrático Médio; $\theta^{\prime}=$ Valor do estimador (Caso Base); $\theta=$ Valor do parâmetro escalar (Simulações); N = Número de Casos;

$$
D(\%)=\frac{\sum_{i}^{N} \frac{F_{c b}-F_{c a}}{F_{c b}}}{N}
$$

Equação 4

Onde: D: Média das Diferenças horárias [\%]; $F_{c a}=$ Fluxo do caso analisado $\left[\mathrm{m}^{3} / \mathrm{s}\right] ; F_{c b}=$ Fluxo do caso base $\left[\mathrm{m}^{3} / \mathrm{s}\right]$;
$\mathrm{N}$ = número de valores analisados, horas do ano, excluindo fluxos negativos.

A análise dos indicadores foi complementada com gráficos de dispersão para obtenção de informações gráficas dos resultados, o que inclui os coeficientes de determinação para verificar a correlação entre dados referenciais.

Em todos os métodos de análise, apenas os fluxos de ar positivos foram selecionados, pois as equações de Maciel (2016) iniciavam com a temperatura de $25^{\circ} \mathrm{C}$ na placa absorvedora, com a obtenção restrita de valores positivos de fluxo. Logo, as temperaturas superficiais inferiores a $25^{\circ} \mathrm{C}$ foram descartadas para evitar fluxos de ar negativos.

\section{Resultados e Discussão}

\section{Caso base}

A partir da simulação, observou-se que a temperatura da placa absorvedora variou de $5,4^{\circ} \mathrm{C}$ a $78,3^{\circ} \mathrm{C}$. Os fluxos resultantes da aplicação da equação de Maciel (2016) sobre esses valores apresentaram alta variação. Foram observados resultados para a ALT1 mínimo de $-0,11 \mathrm{~m}^{3} / \mathrm{s}$ e máximo de $0,09 \mathrm{~m} 3 / \mathrm{s}$, e para ALT2 mínimo de $-0,21 \mathrm{~m}^{3} / \mathrm{s}$ e máximo de $0,17 \mathrm{~m}^{3} / \mathrm{s}$. Porém, como mencionado, os valores de fluxo negativo foram descartados na análise dos resultados.

\section{Casos Analisados}

Como resultado da simulação do Brise-Chaminé-Solar modelado como chaminé térmica, pôde ser observado que houve aumento do fluxo de ar com o aumento da altura da chaminé. Entretanto, não houve fluxos negativos, o que indica que o programa não simulou fluxos descendentes, do meio externo para o interior da chaminé (Tabela 2). Esse resultado pode ser explicado pois o conceito fundamental de uma chaminé solar é utilizar a radiação solar para proporcionar fluxo de ar ascendente. Portanto, se a temperatura do ar de descarga na saída da chaminé for menor que a temperatura do ar ambiente, a chaminé solar é automaticamente desligada no EnergyPlus, uma vez que a temperatura da saída deve ser maior do que a temperatura ambiente para causar fluxo de ar ascendente (LEE; STRAND, 2009). O menor erro absoluto médio (EAM) e erro quadrático médio (EQM) foram obtidos para ALT1 desse modelo, 37,35\% e 0,0004 respectivamente.

O método de zonas térmicas conectadas apresentou EAMs elevados, o que é coerente, visto que os dispositivos modelados como zona térmica não descrevem o efeito chaminé, apenas a diferença de pressão entre as zonas. $\mathrm{O}$ mínimo do EAM foi de 139,15\% (ALT2 - janelas abertas), mais de duas vezes o maior EAM do modelo chaminé térmica. Já o EQM mínimo foi de 0,0017 (ALT1 - janelas fechadas), enquanto o EQM máximo do modelo de 
chaminé térmica foi de 0,0014 (ALT2), o que sugere que o EQM seja um indicador muito sensível para análise dos fluxos de ar de simulações. Entretanto, em todas as opções, foi possível notar diferenças maiores para os dispositivos modelados como zona térmica do que como chaminé térmica, o que confirma a coerência dos resultados.

Embora o destaque seja para o modelo de chaminé térmica, os fluxos obtidos como zonas combinadas apresentou valores na mesma grandeza aos fluxos do modelo de chaminé térmica (Tabela 2), o que indica uma boa coerência entre as simulações. A diferença média horária foi de $102,8 \%$, o que está dentro do intervalo das diferenças médias horárias do modelo de chaminé térmica. Seu EAM de $65,10 \%$ é mais próximo de $51,80 \%$ (modelo chaminé térmica) do que o mínimo de $139,5 \%$ do modelo de zonas térmicas em rede. Apenas o EQM coincidiu com o caso de janelas fechadas do modelo de rede, em que ambos estão submetidos às mesmas condições de infiltração. Deve-se destacar que a grande limitação desse método é o fato do número de zonas ser restrito a duas, o que impossibilitou sua aplicação para a simulação da ALT1.

O resultado da diferença horária média representa que o EnergyPlus subestimou o fluxo de ar em relação ao caso base quando positivo e superestimou quando negativo. Quando consideradas as alturas ALT1 e ALT2, a menor diferença horária ocorreu entre o dispositivo modelado como chaminé térmica e o caso base. Entretanto, a diferença horária média entre o "BCS Modelado como Zonas, janelas fechadas" foi menor que do modelo "BCS Modelado como Chaminé Térmica ALT1". Logo, embora apresentados resultados parciais coerentes, ocorreram limitações na comparação entre fluxos de ar de maneira isolada, restringindo a utilização desse indicador à identificação de que o programa subestima ou superestima os fluxos de ar do caso base comparados ao modelo testado.

Tabela 2 - Indicadores estatísticos aplicados aos casos estudados

\begin{tabular}{|c|c|c|c|c|c|c|c|c|}
\hline & \multirow{2}{*}{\multicolumn{2}{|c|}{$\begin{array}{l}\text { BCS Modelado como } \\
\text { Chaminé Térmica }\end{array}$}} & \multicolumn{4}{|c|}{ BCS Modelado como Zonas Térmicas } & \multirow{2}{*}{\multicolumn{2}{|c|}{$\begin{array}{l}\text { BCS Modelado como Zonas } \\
\text { Combinadas }\end{array}$}} \\
\hline & & & \multicolumn{2}{|c|}{ Janelas Fechadas } & \multicolumn{2}{|c|}{ Janelas Abertas } & & \\
\hline & ALT1 & ALT2 & ALT1 & ALT2 & ALT1 & ALT2 & ALT1 & ALT2 \\
\hline EAM (\%) & 37,35 & 51,80 & 174,25 & 229,05 & 194,25 & 139,15 & - & 65,10 \\
\hline EQM & 0,0004 & 0,0014 & 0,0017 & 0,0049 & 0,0020 & 0,0019 & - & 0,0017 \\
\hline Diferença (\%) & 164,9 & $-72,5$ & 87,5 & $-352,7$ & $-756,0$ & $-352,7$ & - & $-102,8$ \\
\hline Fluxo Máximo $\left(\mathrm{m}^{3} / \mathrm{s}\right)$ & 0,048 & 0,082 & 0,003 & 0,019 & 0,153 & 0,172 & - & 0,079 \\
\hline Fluxo Mínimo (m³/s) & 0,000 & 0,000 & 0,000 & 0,000 & 0,000 & 0,000 & - & 0,000 \\
\hline
\end{tabular}

Fonte: Os autores.

As Figuras 10 e 11 mostram a melhor correlação dos fluxos para o modelo chaminé térmica, identificado em vermelho, cujo coeficiente de determinação para uma equação linear é 0,792 (ALT1) e 0,791 (ALT2). Pôde ser observado que este é o único modelo que apresenta uma correlação aceitável com o caso base.

O modelo de janelas fechadas, identificado em azul, apresentou coeficiente de determinação de 0,696 (ALT1) e 0,789 (ALT2). A explicação para os elevados coeficientes de determinação é pelo fato dos fluxos de ar se aproximarem de zero. O modelo de janelas abertas, identificada em verde e o modelo zonas térmicas combinadas, identificado em roxo, não apresentaram coerência entre os dados, com dispersão elevada e coeficientes de determinação de 0,165 (ALT1) e 0,455 (ALT2) e 0,321 (ALT2).

A análise de dispersão contribuiu para o entendimento qualitativo do tratamento dos dados, tanto pela análise visual quanto pelo coeficiente de determinação, embora este último não indique semelhanças entre os dados, mas o ajuste entre os modelos.
Figura 10 -Fluxos de Ar Simulados comparados ao Caso Base, para ALT1

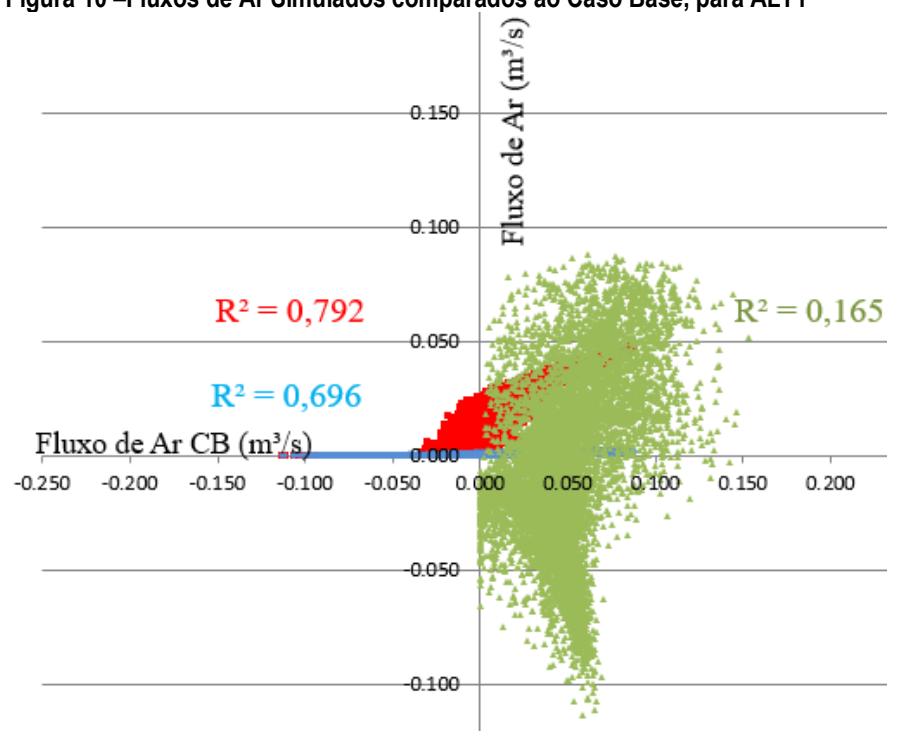

Nota: [vermelho] BCS Modelado como Chaminé Térmica (ALT1); [azul] BCS Modelado como Zonas, Janelas Fechadas (ALT1); [verde] BCS Modelado como Zonas, Janelas Abertas (ALT1). Fonte: Os autores. 


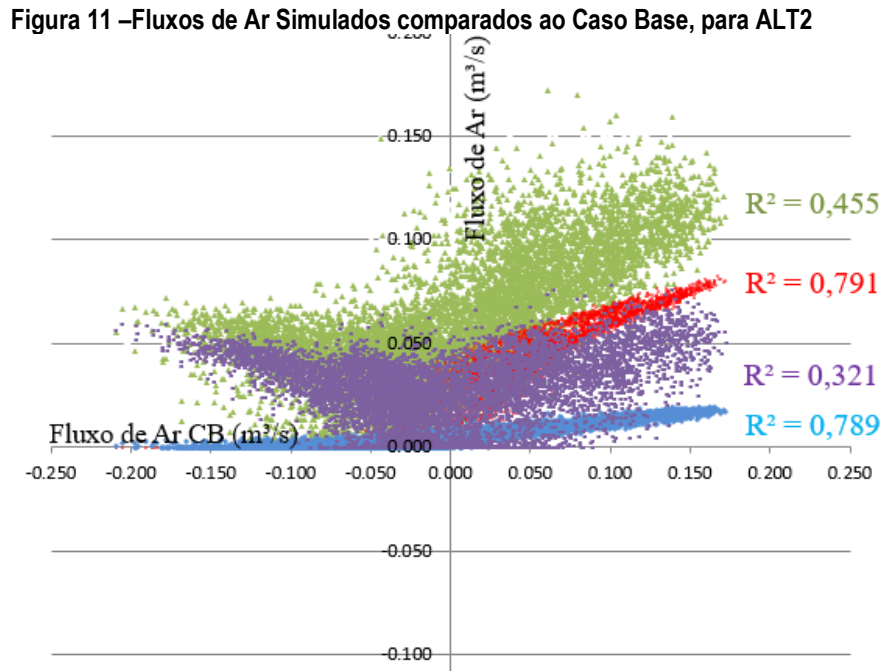

Nota: [vermelho] BCS Modelado como Chaminé Térmica (ALT2); [azul] BCS Modelado como Zonas, Janelas Fechadas (ALT2); [verde] BCS Modelado como Zonas, Janelas Abertas (ALT2); [roxo] BCS Modelado como Zonas Combinadas (ALT2). Fonte: Os autores.

Apesar dos fluxos simulados mais próximos ao caso base apresentarem diferenças cujos EAMs foram $37 \%$ e 52\%, valores relativamente elevados se comparados aos encontrados na literatura por Neves e Roriz (2012), Neves e Silva (2017) e Koronaki (2013), deve-se destacar algumas limitações das simulações. Na simulação foram utilizados materiais idênticos aos do protótipo construído, entretanto, suas propriedades foram baseadas na simulação de Maciel (2016) e na NBR 15220 (ABTN, 2005), assim como o coeficiente de descarga da chaminé solar, estabelecido por Andersen (1995). Além disso, variáveis como a radiação solar, intensidade e direção dos ventos não foram ajustadas de acordo com as condições de medição e simulação CFD de Maciel (2016), uma vez que a simulação foi realizada para todas as horas do ano com um arquivo climático representativo do local. Lee e Strand (2009), ao comparar a eficiência da mesma chaminé solar em duas simulações distintas, um dia e um ano, identificaram redução da eficiência do dispositivo de até 49\% na simulação anual se comparada a de um único dia, pela maior variabilidade meteorológica da primeira condição. Diante das limitações de calibração das variáveis ambientais em uma simulação anual, recomenda-se que, sempre que possível, seja realizado o maior número de calibrações de parâmetros para a obtenção de diferenças entre medição e simulação mais baixas, como apresentado por Neves e Roriz (2012), Neves e Silva (2017) e Koronaki (2013).
Portanto, para esse caso, vê-se que o modelo de chaminé térmica é indicado para a simulação do Brise-ChaminéSolar, cujos resultados de Maciel (2016) o validaram para outros tipos de simulações. Ainda, o EAM e a média das diferenças horárias forneceram indícios para adoção do modelo de zonas combinadas em simulações complexas, em que uma chaminé solar possa ser integrada a outros modelos de ventilação. No entanto, mais estudos são necessários.

\section{Conclusões}

Os resultados obtidos a partir da simulação com o EnergyPlus comprovaram que o modelo de cálculo ThermalChimney é válido para simular os efeitos da ventilação natural em chaminés solares.

O tratamento estatístico com o Erro Absoluto Médio (EAM) e Erro Quadrático Médio (EQM) apresentou resultados coerentes entre o caso base e os demais modelos analisados. Considerou-se EAMs entre $37 \%$ e $52 \%$ e EQMs entre 0,0005 e 0,0015 como máximos aceitáveis para determinar a semelhança entre fluxos de ar em simulações anuais.

Os demais modelos de ventilação foram usados para estabelecer a sensibilidade dos indicadores estatísticos e forneceram parâmetros para análise comparativa de fluxo de ar em base anual. Os valores de EQM apresentaram pequenas diferenças entre todos os casos analisados, de 0,0004 a 0,0049. Já o EAM apresentou diferenças significativas de acordo com os modelos utilizados. Com este indicador, o modelo de cálculo de zonas combinadas chegou a $65 \%$, e mostrou ser válido assim como o modelo de chaminé térmica, embora seu EQM tenha se aproximado dos EQMs do modelo de rede. Este resultado mostra que há indícios da validade do modelo de zonas combinadas, o que precisa ser melhor investigado. Sendo assim, mais estudos são necessários para confirmar os limites recomendados neste trabalho.

Para a análise estatística anual relacionada a fluxos de ar, recomenda-se a utilização do EAM e análise da dispersão dos dados. O EQM e a média das diferenças horárias podem ser utilizados como indicadores complementares, visto que a análise baseada em mais de um indicador contribuiu para aumentar a confiabilidade do tratamento dos dados.

\section{Agradecimentos}

Os autores agradecem o apoio da CAPES e da FAPEMIG. 


\section{Referências}

ABNT - ASSOCIAÇÃO BRASILEIRA DE NORMAS TÉCNICAS. NBR 15220-3: Desempenho térmico de edificações - parte 3: Zoneamento bioclimáticos brasileiros e diretrizes construtivas para habitação unifamiliar de interesse social. Rio de Janeiro, 2005.

ANDERSEN, K. Theoretical considerations on natural ventilation by thermal buoyancy. In: Annual meeting of the American Society of Heating, Refrigeration and Air-Conditioning Engineer, 1995, San Diego. Proceedings... San Diego: ASHRAE Transactions, 1995.

ASHRAE - AMERICAN SOCIETY OF HEATING, REFRIGERATING AND AIR-CONDITIONING ENGINEERS. Standard 140: Standard method of test for the evaluation of building energy analysis computer programs. Atlanta, 2011.

ASADI, S.; FAKHARI, M.; FAYAZ, R.; MAHDAVIPARSA, A. The effect of solar chimney layout on ventilation rate in buildings. Energy and Buildings, v. 123, p. 71-78, jul. 2016. doi:https://doi.org/10.1016/j.enbuild.2016.04.047

CHEN, Q. Ventilation performance prediction for buildings: A method overview and recent application. Building and Environment, v. 44, p. 848-858, abr. 2009. doi:https://doi.org/10.1016/j.buildenv.2008.05.025

ENERGYPLUS. EnergyPlus documentation: Engineering Reference: the reference to EnergyPlus calculation. US Department of Energy, 2015. Disponível em: <https://energyplus.net/sites/default/files/pdfs_v8.3.0/EngineeringReference.pdf> Acesso em: 25.05.2018.

GUIMARÃES, I.; CARLO, J. Caracterização bioclimática da cidade de Viçosa-MG. In: ENCONTRO NACIONAL DE CONFORTO NO AMBIENTE CONSTRUÍDO, 11., 2011, Armação de Búzios. Anais... Armação de Búzios: ENCAC, 2011. Disponível em: http://www.infohab.org.br/encac/files/2011/Top4art29.pdf. Acesso em: Acesso em: 25.05.2018.

HOSIEN, M.; SELIM, S. Effects of the geometrical and operational parameters and alternative outer cover materials on the performance of solar chimney used for natural ventilation. Energy and Buildings, v. 138, p. 355-367, mar. 2017. doi:https://doi.org/10.1016/j.enbuild.2016.12.041

IMRAN, A.; JALIL J.; AHMED, S. Induced flow for ventilation and cooling by a solar chimney. Renewable Energy, v.78, p. 236244, jun. 2015. doi:https://doi.org/10.1016/j.renene.2015.01.019

KORONAKI, P. The impact of configuration and orientation of solar thermosyphonic systems on night ventilation and fan energy savings. Energy and Buildings, v. 57, p. 119-131, fev. 2013. doi:https://doi.org/10.1016/j.enbuild.2012.10.054

LEE, K.; STRAND, R. Enhancement of natural ventilation in buildings using a thermal chimney. Energy and Buildings, v. 41, p. 615-621, jun. 2009. doi:https://doi.org/10.1016/j.enbuild.2008.12.006

MACIEL, L. Desenvolvimento de dispositivo de proteção solar com ênfase na ventilação natural: Um método de avaliação baseado em CFD. 2016. Dissertação (Mestrado em Arquitetura e Urbanismo) - Universidade Federal de Viçosa, Viçosa, 2016.

MElO, A.; WeStPhAl, F.; MATOS, M. Apostila do Curso Básico do Programa Energyplus. Laboratório de Eficiência Energética em Edificações. Florianópolis, 2009. Disponível em:

http://www.labeee.ufsc.br/sites/default/files/disciplinas/ECV4202_Apostila_EnergyPlus_0.pdf. Acesso em: 25.05.2018.

NEVES, L.; RORIZ, M. Procedimentos estimativos do potencial de uso de chaminés solares para promover a ventilação natural em edificações de baixa altura. Ambiente Construído, v. 2, n. 1, p. 177-192, jan./mar. 2012. doi:http://dx.doi.org/10.1590/S167886212012000100012

NEVES, L.; SILVA, S. Analise paramétrica de chaminés solares visando à otimização de desempenho em climas típicos do território brasileiro. Ambiente Construído, v.17, n. 1, p. 163-182, jan./mar. 2017. doi http://dx.doi.org/10.1590/s167886212017000100130.

SCHULZE, T.; EICKER, U. Controlled natural ventilation for energy efficient buildings. Energy and Buildings, v. 56, p. 221-232, jan. 2013. doi:https://doi.org/10.1016/j.enbuild.2012.07.044

SHI, L.; ZHANG, G. An empirical model to predict the performance of typical solar chimneys considering both room and cavity configurations. Building and Environment, v. 103, p. 250-261, jul. 2016. doi:https://doi.org/10.1016/j.buildenv.2016.04.024 
SHI, L.; ZHANG, G.; CHENG, X.; GUO, Y.; WANG, J.; CHEW, M. Developing an empirical model for roof solar chimney based on experimental data from various test rigs. Building and Environment, v. 110, p. 115-128, dez. 2016.

doi:https://doi.org/10.1016/j.buildenv.2016.10.002

ZHAI, X.; SONG, Z.; WANG, R. A review for the applications of solar chimneys in buildings. Renewable and Sustainable Energy Reviews, v.15, p. 3757-3667, out. 2011. doi:https://doi.org/10.1016/j.rser.2011.07.013

ZHANG, R.; LAM, K.; YAO, S; ZHANG, Y. Coupled EnergyPlus and computational fluid dynamics simulation for natural ventilation. Building and Environment, v. 68, p. 100-113, out. 2013. doi:https://doi.org/10.1016/j.buildenv.2013.04.002

\section{${ }^{1}$ Matheus Menezes Oliveira}

Arquiteto e Urbanista. Mestre em Arquitetura e Urbanismo. Endereço postal: Av. P. H. Rolfs, s/nº, Centro, Viçosa, Minas Gerais, Brasil, 36570-900

\section{${ }^{2}$ Joyce Correna Carlo}

Arquiteta e Urbanista. Doutora em Engenharia Civil. Endereço postal: Av. P. H. Rolfs, s/nº, Centro, Viçosa, Minas Gerais, Brasil, 36570-900 